\title{
Cross-spectral measurement of neural signal transfer
}

\author{
Mark D. McDonnell ${ }^{a}$, Swaminathan Sethuraman ${ }^{b}$, Laszlo B. Kish ${ }^{b}$, Derek Abbott $^{a}$ \\ ${ }^{a}$ Centre for Biomedical Engineering (CBME) and School of Electrical \& Electronic Engineering, The \\ University of Adelaide, SA 5005, Australia; \\ ${ }^{b}$ Department of Electrical Engineering, Texas A\&M University, College Station, TX 77843-3128, \\ USA
}

\begin{abstract}
The phenomenon of noise enhanced signal transfer, or stochastic resonance, has been observed in many nonlinear systems such as neurons and ion channels. Initial studies of stochastic resonance focused on systems driven by a periodic signal, and hence used a signal to noise ratio based measure for comparison between the input and output of the system. It has been pointed out that for the more general case of aperiodic signals other measures are required, such as cross-correlation or information theoretical tools. In this paper we present simulation results obtained in a model neural system driven by a broadband aperiodic signal, and producing a signal imitating neural spikes. The system is analyzed by using cross-spectral measures.
\end{abstract}

Keywords: noise, cross spectral density, stochastic resonance, SNR, aperiodic stochastic resonance, Wiener filter, coherence function

\section{INTRODUCTION}

This paper aims to build on previous work which introduced a new measure to the study of Stochastic Resonance (SR), that of a frequency dependent Signal to Noise Ratio (SNR), based on the Cross-power Spectral Density (CSD) between input and output signals in a nonlinear system. ${ }^{1,2}$ It was pointed out in this work that conventional measures of the output SNR in systems in which SR occurs fail both for conventional periodic SR, when the signal is large compared to the noise, and for broadband input signals. This is due to the conventional SNR measure depending on measuring the output noise power as that which occurs when no signal is present. However, for more general conditions, the input signal and noise cross-modulate, and the output noise spectrum cannot be taken as that which occurs when no signal is present. Hence, for computation of output SNRs in such systems, it is necessary to be able to separate output signal and noise components for all frequencies at which the signal exists. Hence it was proposed (in particular for the context of SR research) that an appropriate means of achieving this separation is to classify the output signal as that component of the output spectrum which is the magnitude of the CSD between the input signal and the system output, divided by the Power Spectral Density (PSD) of the system input, with the remainder of the output spectrum being noise. Here, we show that such a separation of signal and noise has a strong basis in signal processing theory and applications, and relate it to other standard signal processing techniques, including the coherence function ${ }^{3}$ and Wiener-Kolmogorov filtering. ${ }^{4}$ We illustrate the method can be applied to aperiodic SR in neurons, by examining a model neural system driven by a broadband input signal.

\section{POWER AND CROSS-POWER SPECTRAL DENSITIES}

The PSD, $S_{x x}(f)$, of an ergodic, finite power signal (such as a stationary random signal), $x(t)$, is given by the Fourier transform of its autocorrelation function, ${ }^{3} R_{x x}(\tau)$, as

$$
S_{x x}(f)=\int_{-\infty}^{\infty} R_{x x}(\tau) \exp (-j 2 \pi \tau) d \tau .
$$

Further author information: (Send correspondence to Mark D McDonnell.)

Mark D. McDonnell.: E-mail: mmcdonne@eleceng.adelaide.edu.au, Telephone: +61 883036296 Fax: +61 883034360

Swaminathan Sethuraman.: E-mail: swamijs@ee.tamu.edu

Derek Abbott.: E-mail: dabbott@eleceng.adelaide.edu.au, Telephone: +61 883035748

Laszlo B. Kish.: Email:laszlo.kish@ee.tamu.edu 
The CSD of two such signals, $x(t)$ and $y(t)$ is given by the Fourier transform of the cross correlation of $x$ and $y, R_{x y}(t)$, as

$$
S_{x y}(f)=\int_{-\infty}^{\infty} R_{x y}(\tau) \exp (-j 2 \pi \tau) d \tau .
$$

Unlike the PSD function, which is always real, the CSD is a complex function of frequency. We also state a well known inequality ${ }^{3}$ relating the CSD to the PSDs of $x$ and $y$,

$$
\left|S_{x y}(f)\right|^{2} \leq S_{x x}(f) S_{y y}(f) .
$$

One of the uses for the CSD function, is the coherence function, which for two signals $x(t)$ and $y(t)$ is defined as ${ }^{3}$

$$
\Gamma_{x y}^{2}(f)=\frac{\left|S_{x y}(f)\right|^{2}}{S_{x x}(f) S_{y y}(f)} .
$$

This function is real valued between 0 and 1 , and is a measure of the linearity between $x$ and $y$, i.e. if a perfect linear relationship exists between $x$ and $y$ at frequency $f$ then the coherence function will be equal to one at that frequency and $\left|S_{x y}(f)\right|^{2}=S_{x x}(f) S_{y y}(f)$. Thus, it can be considered to be a correlation coefficient for the frequency domain.

The coherence function has been used previously in the context of SR, both for conventional periodic SR and aperiodic SR. ${ }^{5,6}$ The first work on aperiodic stochastic resonance used the cross-correlation measure. ${ }^{7}$ It was subsequently noted ${ }^{5}$ that if the coherence function can be found, the cross correlation measure and the correlation coefficient easily follows since the correlation between the input and output is

$$
C_{x y}=\int_{f} \operatorname{Re}\left[S_{x y}(f)\right] d f .
$$

The input and output autocorrelation functions, or mean square power's are similarly obtained from the integral over all $f$ of their PSDs. Hence, the correlation coefficient is

$$
\rho_{x y}=\frac{\int_{f} \operatorname{Re}\left[S_{x y}(f)\right] d f}{\sqrt{\int_{f} S_{x x}(f) d f \int_{f} S_{y y}(f) d f}} .
$$

\subsection{Linear systems}

CSDs can arise when two correlated signals are added, for example, if the two signals are $s(t)$ and $n(t)$, the result $x(t)=$ $s(t)+n(t)$ has auto-correlation

$$
\begin{aligned}
R_{x x}(\tau) & =\langle x(t) x(t-\tau)\rangle=\langle[s(t)+n(t)][s(t-\tau)+n(t-\tau)]\rangle \\
& =R_{s s}(\tau)+R_{s n}(\tau)+R_{n s}(\tau)+R_{n n}(\tau) .
\end{aligned}
$$

Taking the Fourier transform of both sides gives the PSD of $x(t)$ in terms of the spectral densities of $s(t)$ and $n(t)$ and the CSD of $s(t)$ and $n(t)$ as

$$
S_{x x}(f)=S_{s s}(f)+2 \operatorname{Re}\left[S_{s n}(f)\right]+S_{n n}(f),
$$

where we have used the property that $S_{s n}(f)=S_{n s}^{*}(f)$, where $*$ is the complex conjugate. Note that if $s(t)$ and $n(t)$ were uncorrelated then $S_{s n}(f)$ is zero for all $f$ and the output spectral density is the sum of the spectral densities of $x(t)$ and $n(t)$.

We may also be interested in the CSD of $s(t)$ and $x(t)$, i.e. $S_{s x}(f)$. This is, again, the Fourier transform of the cross correlation of $s(t)$ and $x(t)$, which is

$$
\begin{aligned}
R_{s x}(\tau) & =\langle s(t) x(t-\tau)\rangle=\langle s(t)[s(t-\tau)+n(t-\tau)]\rangle \\
& =R_{s s}(\tau)+R_{s n}(\tau) .
\end{aligned}
$$

Taking the Fourier transform of both sides gives

$$
S_{s x}(f)=S_{s s}(f)+S_{s n}(f) .
$$


The magnitude squared of $S_{s x}$ is given by

$$
\begin{aligned}
\left|S_{s x}(f)\right|^{2} & =S_{s x}(f) S_{s x}^{*}(f) \\
& =\left(S_{s s}(f)+S_{s n}(f)\right)\left(S_{s s}(f)+S_{s n}(f)\right)^{*} \\
& =S_{s s}(f)\left(S_{s s}(f)+2 \operatorname{Re}\left[S_{s n}(f)\right]\right)+\left|S_{s n}(f)\right|^{2} .
\end{aligned}
$$

If we substitute (2) into (3) we get

$$
\left|S_{s x}(f)\right|^{2}=S_{s s}(f)\left(S_{x x}(f)-S_{n n}(f)\right)+\left|S_{s n}(f)\right|^{2}
$$

and the coherence function is

$$
\Gamma_{s x}^{2}(f)=1-\frac{S_{n n}(f)}{S_{x x}(f)}+\frac{\left|S_{s n}(f)\right|^{2}}{S_{s s}(f) S_{x x}(f)} \forall f \text { s.t } S_{s s}(f) S_{x x}(f) \neq 0 .
$$

\subsubsection{Uncorrelated signal and noise}

If the signal and noise are uncorrelated then $S_{s n}(f)=0$ and from (2) the sum of the PSD's is the output PSD. Therefore from (4) we get

$$
\left|S_{s x}(f)\right|^{2}=S_{s s}^{2}(f)
$$

and the coherence function between $s$ and $x$ is

$$
\Gamma_{s x}^{2}(f)=\frac{S_{s s}(f)}{S_{s s}(f)+S_{n n}(f)},
$$

and is only unity when noise is absent. Thus, the coherence function can be reduced from unity by the presence of noise, even when the signal and noise are added and uncorrelated, simply due to the noise being nonzero at frequencies present in the signal.

\subsection{Nonlinear systems}

We now consider the general case of a system with input signal, $s(t)$, subject to additive noise, $n(t)$, so that the overall input is $x(t)=s(t)+n(t)$. Let the output be the result of a nonlinear transformation of $x(t)$, i.e. $y(t)=T[x(t)]$. Then the PSDs of $s$ and $y$ are $S_{s s}(f)$ and $S_{y y}(f)$ respectively, and $S_{s y}(f)$ is the CSD between $s$ and $y$.

An ideal metric for such a system is one which will give a consistent, frequency dependent, phase independent measure for an output SNR under all circumstances, including either periodic, aperiodic or broadband input signals, and regardless of whether the signal is subthreshold or not, or has small amplitude compared to the noise or not. To this end Kiss ${ }^{1}$ previously defined the following "generalized amplification" factor, which is a complex function of frequency,

$$
K(f)=\frac{S_{s y}(f)}{S_{s s}(f)}
$$

Using carets to indicate output signal and noise, as opposed to input signal and noise, we can simplify the notation used by Kiss ${ }^{1}$ to define the "generalized output signal" spectrum as

$$
\hat{S}_{s s}(f)=S_{s s}(f)|K(f)|^{2}=\frac{\left|S_{s y}(f)\right|^{2}}{S_{s s}(f)} .
$$

Note that since the PSD is a real number, $\left|S_{s s}(f)\right|^{2}$ is simply $S_{s s}(f)^{2}$. It was noted that $\hat{S}_{s s}(f)$ can be considered to be the spectrum of the signal component of the output signal and that the remainder of the output spectrum is noise so that

$$
\hat{S}_{n n}(f)=S_{y y}(f)-\hat{S}_{s s}(f) .
$$

Substituting (7) into (8) gives the output noise spectrum in terms of the signal PSD, the CSD and the overall output PSD as

$$
\hat{S}_{n n}(f)=S_{y y}(f)-\frac{\left|S_{s y}(f)\right|^{2}}{S_{s s}(f)} .
$$


Thus, the output SNR obtained with these quantities is a function of frequency given by

$$
\operatorname{SNR}(f)=\frac{\frac{\left|S_{s y}(f)\right|^{2}}{S_{s s}(f)}}{S_{y y}(f)-\frac{\left|S_{s y}(f)\right|^{2}}{S_{s s}(f)}} .
$$

We now add several observations and caveats to this measure.

Firstly, in order to avoid infinities, the quantity $K(f)$, and therefore the output SNR, must be defined only for frequencies for which $S_{s s}(f)$ is non zero and not a delta function. Delta functions will occur in the input spectrum if the input has non zero mean, or contains periodic components. To avoid this scenario, we will consider only non-periodic, zero mean, ergodic random signals.

Secondly, the "generalized amplification" factor can be seen to look like the same quantity as the susceptibility function, $\chi(f)$ used in the context of linear response theory, for which it is stated that

$$
S_{s y}(f)=\chi(f) S_{s s}(f) .
$$

Linear response theory also states that for a sufficiently weak input signal, the output spectrum is

$$
S_{y y}(f)=S_{y y}^{0}(f)+|\chi(f)|^{2} S_{s s}(f),
$$

where $S_{y y}^{0}(f)$ is the output PSD which occurs when $s(t)=0 .{ }^{5}$ However, for the general case we are considering, these equations are not valid, since in linear response theory, the susceptibility is taken to be constant for a system, regardless of the input, whereas $K(f)$ is defined for a combination of the system and input.

Thirdly, from (1), we can rewrite (7) and (8) in terms of the coherence function as

$$
\hat{S}_{s s}(f)=\Gamma_{s y}^{2}(f) S_{y y}(f)
$$

and

$$
\hat{S}_{n n}(f)=\left(1-\Gamma_{s y}^{2}(f)\right) S_{y y}(f) .
$$

Hence, if $s$ and $y$ were linearly related, then $1-\Gamma_{s y}^{2}(f)=0$, the output signal would be the entire output spectrum, and the output noise would be zero. We also can now write

$$
\operatorname{SNR}(f)=\frac{\Gamma_{s y}^{2}(f)}{1-\Gamma_{s y}^{2}(f)} .
$$

This relation between frequency dependent SNR and the coherence function can also be found in Gabbiani ${ }^{8}$ and Borst $e t$ $a l,{ }^{9}$ as well as the same means of output signal and noise separation. In particular, it has been applied to neural systems, as early as 1991 by Bialek et al. ${ }^{10}$ It can also be seen that this formulation of SNR is effectively a renormalization of the coherence function. If the coherence function approaches unity, then the SNR approaches infinity. If the coherence function is zero, then the SNR is zero. If the coherence function is one half, then the SNR is unity. Hence, measuring system performance by the SNR gives exactly the same information as the coherence function, and vice versa. However the SNR may be more useful if comparing two systems with nearly the same performance, as the coherence function near zero and one is effectively highly compressed compared to the SNR.

The very fact that the SNR can be expressed in terms of the coherence function, which is a measure of the linearity between two signals, may seem surprising since we have aimed to derive a measure that is robust to nonlinear transformations of a signal. However, the root of this fact can be seen by re-examination of (6) and (7). Even though $K(f)$ may not be a linear function of $f$, these equations define a linear relationship between the PSDs of $s(t)$ and $y(t)$, in the sense that $S_{s y}(f)$ is a linear function of $S_{s s}(f)$. Furthermore, this does not mean that the method is not applicable for highly nonlinear signal transfer, since we do not define $K(f)$ as being applicable for all possible input signal and noise combinations, but define it, given a signal and noise combination.

Fourthly, we note that electronic engineers also use (6) in linear systems theory, except that $K(f)$ is usually specified as the "transfer function," $H(f)$, which is used to define a linear system. Again, the approach taken here differs from such linear systems theory, in that $K(f)$ is not defined by only the system, but also by the input signal and noise. 


\subsection{Applicability to linear systems}

To illustrate that the frequency dependent SNR is applicable to linear systems, we find from Section 2.1 that when two signals $s(t)$ and $n(t)$ are added,

$$
\hat{S}_{s s}(f)=\frac{\left|S_{s x}(f)\right|^{2}}{S_{s s}(f)}=S_{x x}(f)-S_{n n}(f)+\frac{\left|S_{s n}(f)\right|^{2}}{S_{s s}(f)}=S_{s s}(f)+2 \operatorname{Re}\left[S_{s n}(f)\right]+\frac{\left|S_{s n}(f)\right|^{2}}{S_{s s}(f)},
$$

and

$$
\hat{S}_{n n}(f)=S_{n n}(f)-\frac{\left|S_{s n}(f)\right|^{2}}{S_{s s}(f)} .
$$

If the signal and noise are completely uncorrelated then $S_{s n}(f)$ is zero and the output signal and noise are simply the input signal and noise. Thus the SNR of (9) is simply the conventional linear systems definition,

$$
\operatorname{SNR}(f)=\frac{S_{s s}(f)}{S_{n n}(f)}
$$

Notice also that the coherence function is given as in (5) and substitution of this expression into (10) gives exactly (11).

\section{NEW INTERPRETATION}

The quantity labelled as the "generalized output signal," i.e. $\hat{S}_{s s}(f)$, can be interpreted as an estimate for the input signal PSD. The process of obtaining $\hat{S}_{s s}(f)$ is implicitly a linear filtering operation, which depends on knowledge of the input PSD. Hence, this filtering is similar to matched filtering, which also depends on knowledge of the input signal. To illustrate, consider the system as a "black-box," which has as input a known input signal, $s(t)$, and an output $y(t)$. The processes internal to the black box are the addition of the input noise, $n(t)$, to $s(t)$ and the nonlinear transformation, $T[s(t)+n(t)]$. To obtain $\hat{S}_{s s}$, the CSD of the output of the black-box, and the input signal, $s(t)$ is found, and divided by the PSD of the input signal. These two operations form the filtering. The SNR can then be found from (9).

It is evident that such a filter will not be useful if the input signals's PSD is unknown, since then there is no means of obtaining $\hat{S}_{s s}$ and therefore the output SNR cannot be determined. However, this method can provide a useful means of characterizing a system, and determining performance for an expected input signal.

We note that this formulation has many similarities to the Wiener-Kolmogorov filter, ${ }^{4}$ where the output time signal, $y(t)$ is convolved with the linear filter given by

$$
K(\tau)=F^{-1}\left(\frac{S_{s y}^{*}(f)}{S_{y y}(f)}\right)
$$

This means that in the frequency domain

$$
\hat{S}_{s s}(f)=\frac{S_{s y}^{*}(f)}{S_{y y}(f)} .
$$

Such a technique is well known in signal processing, and has been applied frequently in the computational neuroscience literature in the analysis of neural spike trains. ${ }^{8,11,12}$

Note that if we were to define the spectrum of the output signal as

$$
\hat{S}_{s s}(f)=\frac{\left|S_{s y}(f)\right|^{2}}{S_{y y}(f)}
$$

then the noise spectrum at the output can be considered as the difference between the input spectrum and the output spectrum, which is

$$
\hat{S}_{n n}(f)=S_{s s}(f)-\hat{S}_{s s}(f)=S_{s s}(f)-\frac{\left|S_{s y}(f)\right|^{2}}{S_{y y}(f)},
$$

and the signal to noise ratio at the output is

$$
\operatorname{SNR}(f)=\frac{\frac{\left|S_{s y}(f)\right|^{2}}{S_{y y}(f)}}{S_{s s}(f)-\frac{\left|S_{s y}(f)\right|^{2}}{S_{y y}(f)}}=\frac{\Gamma_{s y}^{2}(f)}{1-\Gamma_{s y}^{2}(f)},
$$

which is precisely the same formula as (10). 


\section{RESULTS}

We wish to apply the generalized frequency dependent SNR formula to a model neural system driven by a broadband and aperiodic input signal. We consider only a simple model of a neuron that encapsulates its main nonlinearity- that of a threshold. The output from the neuron is a spike when the input, $s(t)$, is greater than the neuron's threshold, $\theta$. We also assume additive Gaussian white noise, $n(t)$, is inherent at the input to the neuron, so that the output contains noise due to both the nonlinearity and the input noise. Thus, assuming an infinitesimal refractory, the neuron's output is

$$
y(t)= \begin{cases}1 & \text { if } s(t)+n(t)>\theta \\ 0 & \text { otherwise }\end{cases}
$$

In the remainder of this section we examine the use of the frequency dependent SNR formula of (10) by simulating input and output signals from this model neuron. Note that care must be taken when attempting to calculate spectral densities and SNR's by simulation. Due to the necessity to simulate signals by discrete time series, the Discrete Fourier Transform (DFT) must be used, usually using the Fast Fourier Transform (FFT) algorithm. Inherent in the FFT is a gain factor which depends on the number of samples used in the FFT. Furthermore, unlike for finite energy signals such as pulses, for random signals, the magnitude of the FFT is not necessarily the correct quantity to take as the PSD. Instead, estimation techniques such as Welch's averaged, modified periodogram method are required.

\subsection{Broadband random input signal}

Here we examine the frequency dependent SNR measure for a wideband random input signal. The simplest way to illustrate the effectiveness of the SNR measure is to let the input signal be a bandpass Gaussian random signal, $s(t)$, with zero mean and unity variance. We arbitrarily set the bandwidth to be $2 \mathrm{kHz}$ between 4 and $6 \mathrm{kHz}$. In our simulations we use a sampling frequency of $20 \mathrm{kHz}$. A realization of $s(t)$ is shown in Fig. 1, and its PSD in Fig. 2.

Let the noise, $n(t)$, be Gaussian white noise (in this case, since our simulations have a sampling frequency of 20 $\mathrm{kHz}$, the noise bandwidth is effectively the Nyquist frequency of $10 \mathrm{kHz}$ ). Let the neuron's threshold be $\theta=3$. Hence, since the signal has unity variance, it will nearly always be subthreshold, and hence our simulation is exactly that which is most commonly used in threshold-based SR studies; that of a subthreshold signal which to which noise is added, in order to induce threshold crossings. ${ }^{13,14}$ To illustrate the various spectra involved, Fig. 3 shows plots of the input and output power spectral densities, and the magnitude of the cross spectral density obtained for 1000000 samples, and a noise variance of 4 . It turns out that this is close to the optimal value of noise variance for this signal and threshold. Despite this, only a very slight increase in output PSD is evident in the bandpass frequencies, however, the magnitude of the CSD is relatively large when compared to non-optimal values of noise, and it is this fact which leads to the SNR containing a maximum near this point, as we shall now see.

Fig. 4 shows the SNR of (10) plotted against frequency for a number noise intensities. It is clear that there must be a nonzero value of noise intensity which provides the optimal output response. Since Fig. 4 indicates that the output SNR is about constant for the pass band frequencies, we show in Fig. 5 the value of the output SNR as a function of noise standard deviation, for the center frequency of $5 \mathrm{kHz}$. 


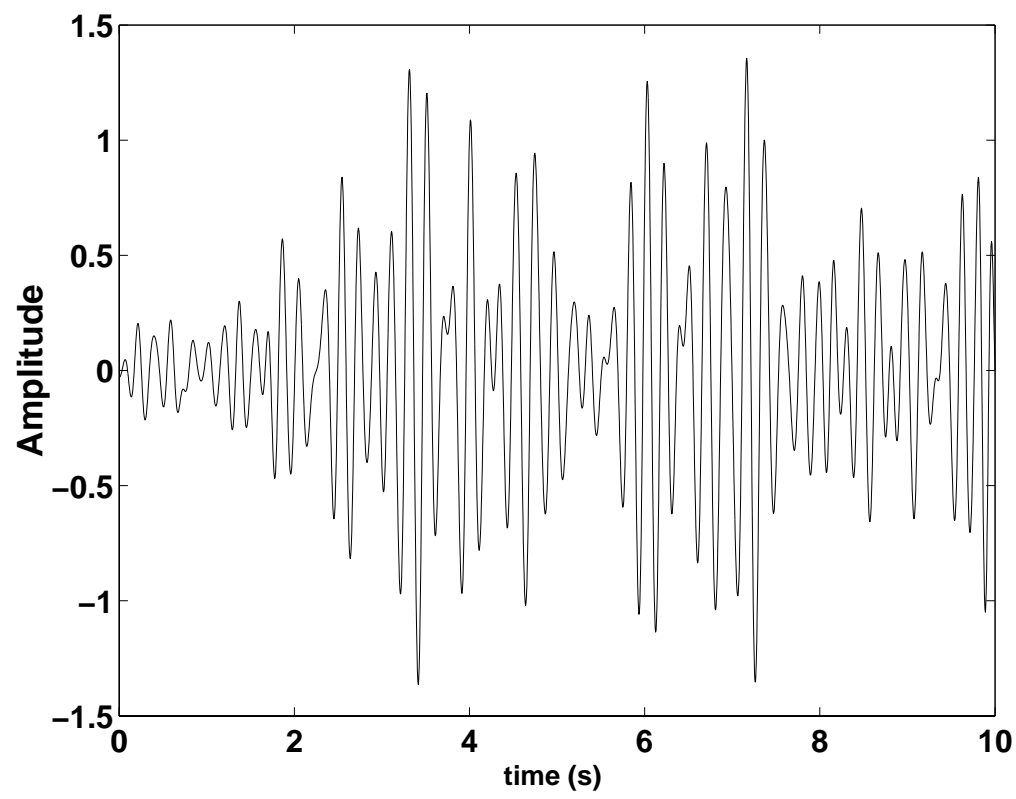

Figure 1. 10 second realization of Gaussian bandpass signal generated by applying an elliptic filter to a sequence of Gaussian distributed samples. Bandwidth is $2 \mathrm{kHz}$ between 4 and $6 \mathrm{kHz}$.

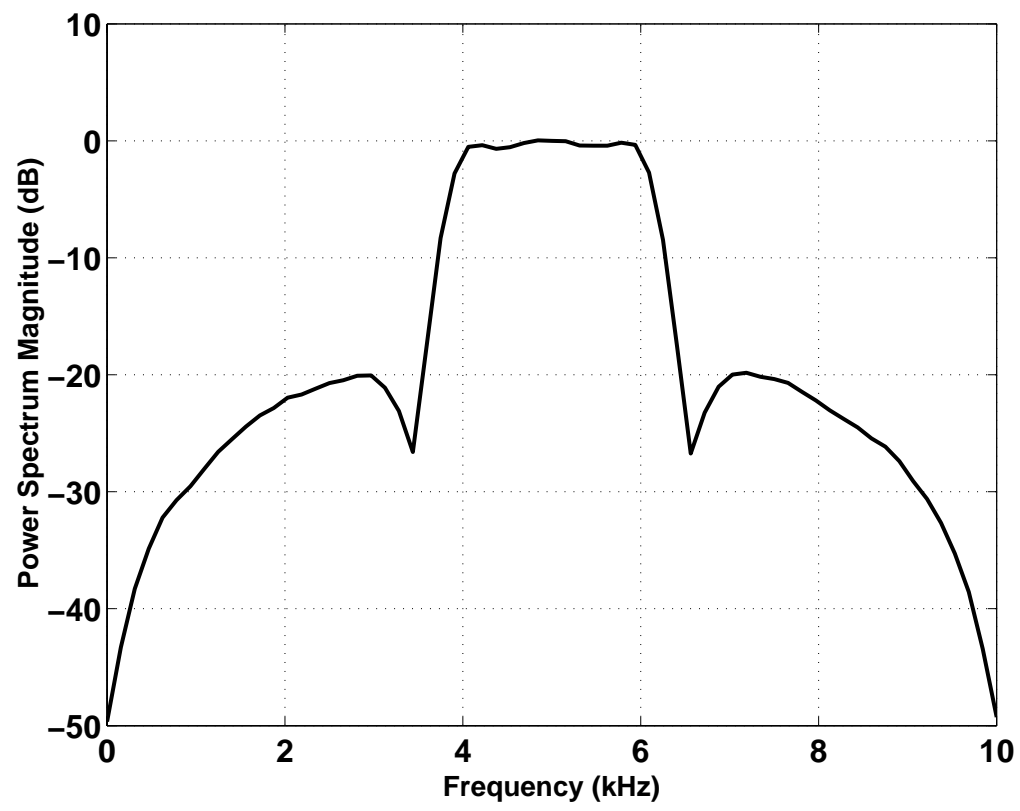

Figure 2. PSD of bandpass signal, implemented using Welch's averaged, modified periodogram method. The bandpass nature of the signal is clearly evident. 


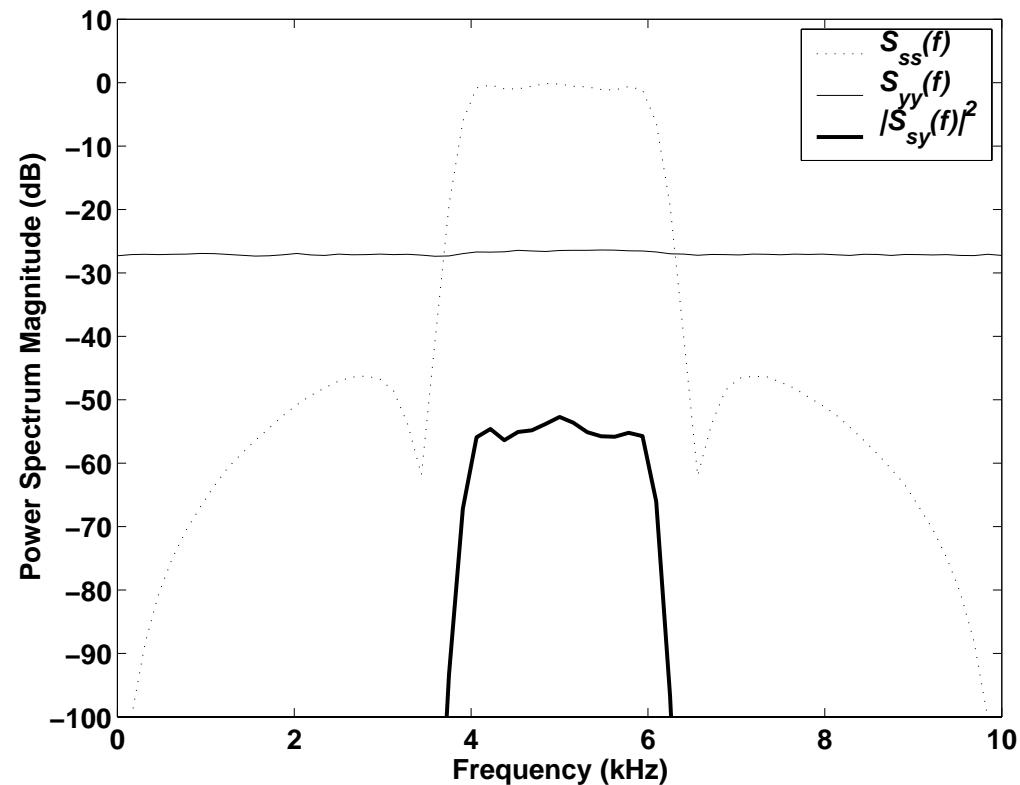

Figure 3. PSD of input and output signals, and magnitude of CSD of input and output signals.

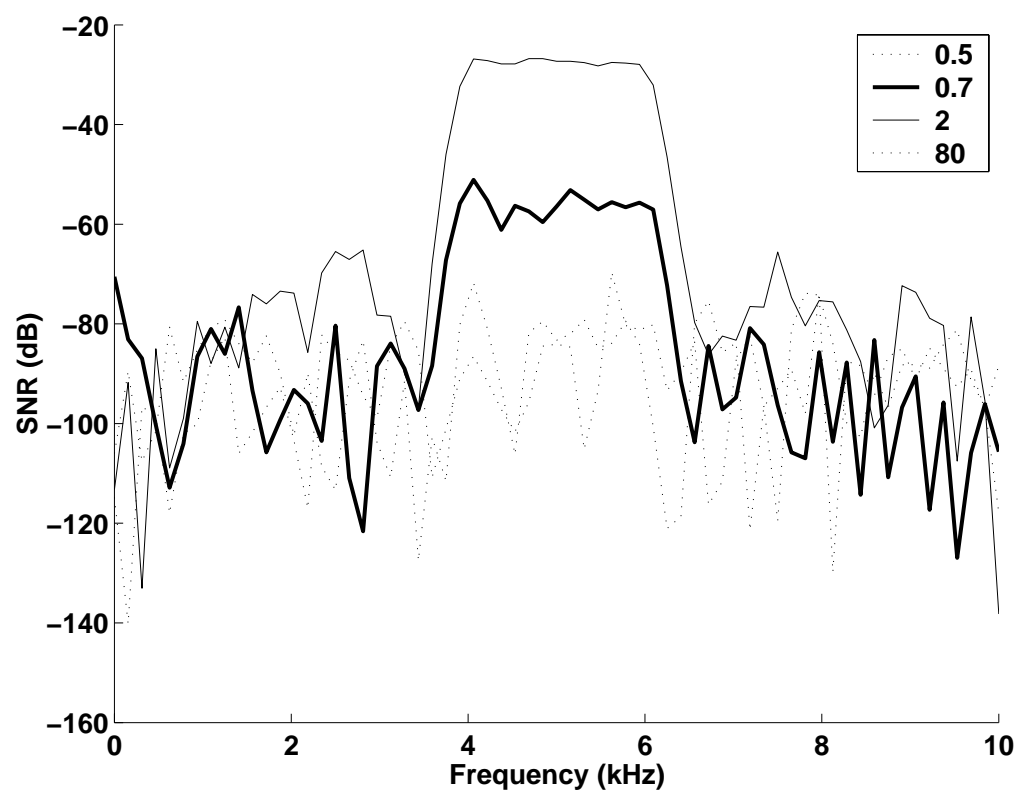

Figure 4. Output SNR against frequency for four values of noise standard deviation. 


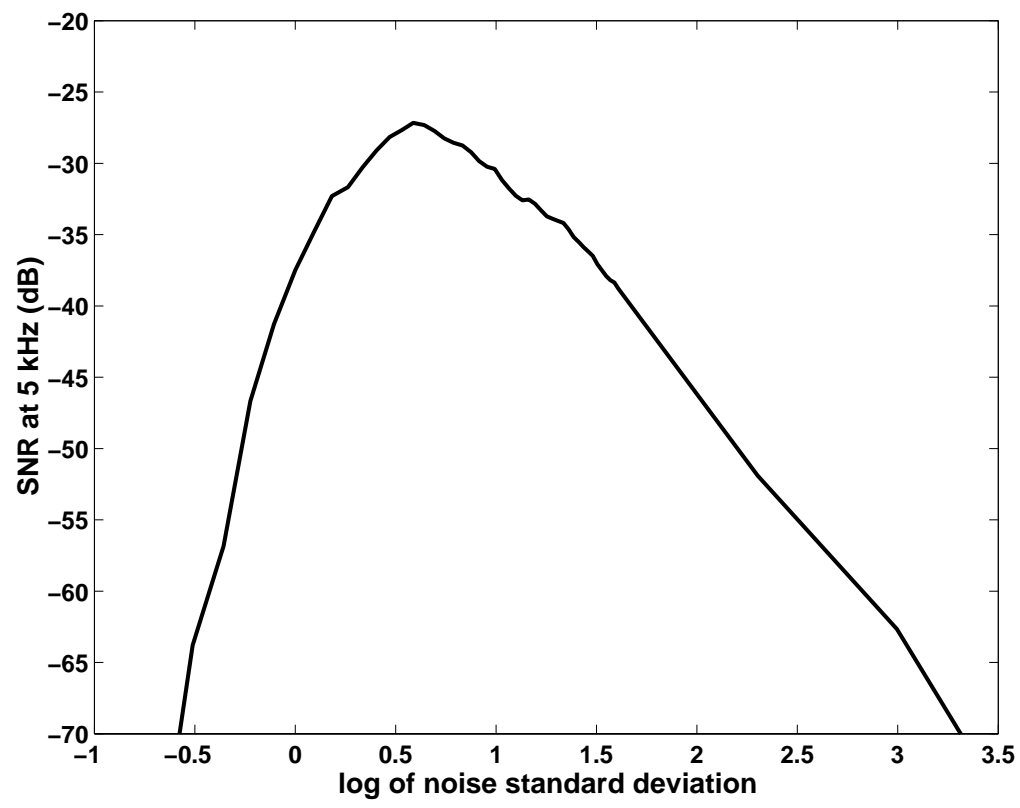

Figure 5. Output SNR at $5 \mathrm{kHz}$ against log of noise standard deviation. The optimal noise standard deviation is 1.8.

\section{CONCLUSIONS}

It is clear from the results of the previous section that although we have shown SR occurring as expected, the very low maximum output SNR means that the output signal is only slightly coherent with the input signal. However for other forms of input signal we would expect the output SNR to be much higher than this. ${ }^{2}$ The main conclusion to be drawn from these simulations is that the frequency dependent output SNR formulation outlined quite clearly is a valid measure for studying SR. We have shown that it is closely related to the coherence function, and Wiener filtering, and since the latter technique has been applied extensively in studies of neural spike trains, we expect that it could be usefully applied to studies of SR in more realistic neural systems than that considered here.

\section{ACKNOWLEDGMENTS}

This work was funded by the Australian Research Council and The Cooperative Research Centre for Sensor, Signal and Information Processing.

\section{REFERENCES}

1. L.B. Kiss. Possible breakthrough: significant improvement of signal to noise ratio by stochastic resonance. In R. Katz, editor, Chaotic, Fractal and Nonlinear Signal Processing, volume 375 of American Institute of Physics Conference Proceedings, pages 382-396, 1996.

2. S. Sethuraman and L.B. Kish. Cross spectra measure of neural signals and noise. In S. M. Bezrukov, H. Frauenfelder, and F. Moss, editors, Fluctuations and Noise in Biological and Biomedical Systems, volume 5110 of Proceedings of SPIE, pages 244-251, 2003.

3. J.S. Bendat. Nonlinear System Techniques and Applications. John Wiley and Sons, Inc, New York, 1998.

4. H.V. Poor. An Introduction to Signal Detection and Estimation. Springer-Verlag, New York, 2nd edition, 1994.

5. A. Neiman, L. Schimansky-Geier, and F. Moss. Linear response theory applied to stochastic resonance in models of ensembles of oscillators. Physical Review E, 56:R9-R12, 1997.

6. V.S. Anishchenko, A.B. Neiman, F. Moss, and L. Schimansky-Geier. Stochastic resonance: noise enhanced order. Uspekhi Fizicheskikh Nauk, 169:7-38, 1999. 
7. J.J. Collins, C.C. Chow, and T.T. Imhoff. Aperiodic stochastic resonance in excitable systems. Physical Review E, 52:R3321-R3324, 1995.

8. F. Gabbiani. Coding of time-varying signals in spike trains of linear and half-wave rectifying neurons. Network: Computation in Neural Systems, 7:61-85, 1996.

9. A. Borst and F.E. Theunissen. Information theory and neural coding. Nature Neuroscience, 2:947-957, 1999.

10. W. Bialek, F. Rieke, R.R. De Ruyter van Steveninck, and D. Warland. Reading a neural code. Science, 252:18541857, 1991.

11. A. Manwani and C. Koch. Synaptic transmission: An information-theoretic perspective. In M. Jordan, M. Kearns, and S. Solla, editors, Advances in Neural Information Processing Systems 10, 1998.

12. A. Manwani and C. Koch. Detecting and estimating signals in noisy cable structures, II: Information theoretical analysis. Neural Computation, 11:1831-1873, 1999.

13. P. Jung and G. Mayer-Kress. Stochastic resonance in threshold devices. Il Nuovo Cimento, 17:827-834, 1995.

14. Z. Gingl, L.B. Kiss, and F. Moss. Non-dynamical stochastic resonance: Theory and experiments with white and arbitrarily coloured noise. EuroPhysics Letters, 29:191-196, 1995. 